\title{
Evaluating a website to teach children safety with dogs
}

\author{
David C Schwebel, ${ }^{1}$ Leslie A McClure, ${ }^{1}$ Joan Severson ${ }^{2}$
}

${ }^{1}$ University of Alabama at Birmingham, Birmingham, Alabama, USA

${ }^{2}$ Digital Artefacts, LLC, lowa City, lowa, USA

\section{Correspondence to} Dr David C Schwebel, Department of Psychology, University of Alabama at Birmingham, 1300 University Blvd, CH 415, Birmingham AL 35294, USA;

schwebel@uab.edu

Received 29 April 2014 Accepted 8 May 2014 Published Online First 28 May 2014
CrossMark

To cite: Schwebel DC, McClure LA, Severson J. Inj Prev 2015;21:e2.

\section{ABSTRACT}

Background Paediatric dog bites are a significant public health problem worldwide. Existing prevention programmes focused on altering children's risky behaviour with pet dogs tend to be atheoretical and only moderately effective.

Objective Test efficacy of a website to train young children in relevant cognitive skills to be safe with pet dogs in their home.

Setting Birmingham, Alabama, USA.

Methods A randomised trial will be conducted with an expected sample of two groups of 34 children (total $\mathrm{N}=68$ ) ages $4-6$ years. One group will engage in the newly designed website at home for 2 weeks and the other group will engage in a control website on transportation safety for an equivalent amount of time. All participants will complete a battery of laboratorybased tests to assess safety with dogs and cognitive functioning at baseline and postintervention.

Outcome measures Primary analyses will be conducted through linear mixed models testing change over time. Children's cognitive functioning, knowledge about safety with dogs, and behaviour with dogs in simulation and in vivo will serve as the primary outcomes.

Clinical trial registration This study is exempt from registry at the US government website, http://www. clinicaltrials.gov, based on being a behavioural trial in the early phases of testing.

This protocol describes an US government-funded randomised trial evaluating the efficacy of a newly developed website to teach young children cognitive skills relevant to behaving safely with pet dogs in the home. Below, we outline the rationale for the research and then present research hypotheses and methods.

\section{STUDY RATIONALE}

\section{Public health significance}

Dog bites are a significant public health problem worldwide. In the USA, the Centers for Disease Control estimates almost five million dog bites occur annually, ${ }^{1}$ resulting in about 800000 individuals requiring medical treatment, 6000 hospitalisations and a dozen deaths. ${ }^{1}{ }^{2}$ Although dog bites affect people at all ages, for several reasons children suffer the highest risk by a large margin. ${ }^{2}{ }^{3}$ First, children behave in unpredictable and active ways, ${ }^{4}$ stressing dogs and sometimes causing animal aggression. Second, children lack the cognitive skills in impulse control, perspective-taking and attention to details needed to recognise, understand and behave appropriately near dogs. Children's misperception, misunderstanding or simple ignorance of a dog's perspective and desires may lead to inappropriate and risky behaviour. Third, children are shorter and weaker than adults, leading to increased prevalence of bites to the head/neck region, which involve greater injury and more substantial treatment than bites to the limbs.

\section{Existing prevention programmes}

Various programmes have been developed to prevent paediatric dog bites. ${ }^{5}$ Early work considered school-based programmes using pre-post or case-control research designs. In one, $3467-$ 8 -year-old children were exposed to a 30 -min classroom lesson. ${ }^{6}$ Several days later, children were observed on the school grounds, where an unfamiliar docile dog was tethered. Children exposed to the lesson behaved more cautiously near the dog than unexposed children. A second study evaluated the BARK (Be Aware, Responsible, and Kind) programme, ${ }^{7}$ which includes an educational workbook, an activity book and a colouring book, all supplemented with a brief video. A pre-post evaluation with almost 500 children (grades 2-4) demonstrated positive changes in knowledge about safe behaviour with dogs, especially among older children. A third study evaluated the Delta DogSafe programme, which used photographs and puppets to tell stories and model safe behaviours for 192 children ages 3-5 years. ${ }^{8}$ In a pre-post experimental design, children exposed to the programme were more likely to recognise photographs of dangerous behaviour with dogs than unexposed children.

More recent research evaluated The Blue Dog, an interactive computer-based programme designed to teach dog safety to children ages 3-6 years. Unlike previous classroom-based interventions, The Blue Dog is an interactive computer-based software programme. Training can be self-initiated and completed at home with a parent. Children and parents consider it entertaining and engaging, ${ }^{9} 10$ qualities that maintain children's interest and encourage them to experience lessons repeatedly and fully.

In The Blue Dog programme, children view multiple animated scenes with child and dog characters, and decide how the child character should interact with the dog in each scene. As an example, in one scene a child approaches a dog sleeping in a basket. The child decides whether to stroke the dog 'good night' or leave the resting dog alone. Incorrect choices (stroking sleeping dog) result in a growling and angry dog character; correct choices yield positive responses from child and dog animations. Empirical testing of The Blue Dog achieved mixed results. ${ }^{9}{ }^{10}$ In one trial of 102 children ages 3-6 years, children completed an eight-item survey 
about safe interaction with dogs prior to and after using The Blue Dog. Children demonstrated a significant increase in knowledge (from about 5.5 to 7.25 out of 8 items correct) after 12-20 min of using the software, and knowledge was retained (especially among older children) over a 2 -week time period during which children used the software two to three times per week at home with parents. ${ }^{9}$ Behaviour change was not assessed. In a second trial, conducted in our own laboratory, 76 children were randomly assigned to use either Blue Dog or a comparison software programme on fire safety at home for 3 weeks. ${ }^{10}$ Recall and recognition of safe behaviour with dogs, plus actual behaviour with an unfamiliar live dog, were assessed prior to and after engagement with the software. Replicating previous results, ${ }^{9}$ children exposed to The Blue Dog showed an increase in recognition knowledge about safe behaviour with dogs. However, children did not spontaneously recall what was safe nor did they actually change their behaviour when faced with an unfamiliar live dog. Taken together, available evidence suggests The Blue Dog is an effective tool to teach children basic knowledge about safety with dogs but it does not appear to yield desired behavioural changes among young children.

\section{THE CURRENT STUDY}

The current study builds from existing intervention programmes -including The Blue Dog-to develop and then evaluate an internet-based child dog bite prevention programme. Existing interventions rely on dog-relevant scenarios and learning by rote rules and/or modelling. These are sensible pedagogical strategies and empirical evidence indicates they convey key knowledge to children but may not result in changed behaviour with dogs.

The present proposal moves beyond the two predominant current strategies for teaching dog safety, learning of rules about safety and modelling of safe behaviour. We believe children also need specific cognitive skills to develop safe behaviour with dogs. In particular, three cognitive abilities-impulse control, perspective taking and attention to details-that typically develop during the preschool years are critical to yield safer behaviour with dogs. We also believe behaviour change strategies must be incorporated into an effective intervention. Children (and their parents) must perceive personal vulnerability for bites, recognise normative behaviour for protection and have motivation for behavioural change. Our proposed intervention teaches cognitive skills and works towards health behaviour change along with offering opportunity for children to learn rules and model safe behaviour.

We propose an internet-based training programme for young children. Today's preschoolers are frequent users of the internet, ${ }^{11}$ and we will train them where many are accustomed to learning-on computer and tablet platforms. We will engage the children with entertaining but educational activities and videos delivered via a medium comfortable to their generation. We will translate our basic science knowledge and theory to a widely accessible public health intervention.

The research includes two phases: (A) develop an interactive, engaging and educational website to train children ages 4-6 years in safe interaction with dogs, and (B) conduct an initial evaluation of the website's usability and training efficacy. We have two specific aims.

First, we plan to develop an innovative and engaging website to train young children in safety with dogs. Today's preschoolers are computer-savvy ${ }^{11}$ and benefit from educational websites that are interactive, entertaining and engaging. ${ }^{12}$ We will develop a website that meets these criteria through interactive games, brief videos, humour and entertainment, and a 'points' system that motivates children to engage in the website repeatedly.

Second, we will evaluate the usability and efficacy of the website. We will conduct a two-group randomised trial with premeasurement and postmeasurement to investigate four outcomes: (2A) usability of the website, (2B) efficacy of the programme to improve children's relevant cognitive skills, (2C) efficacy to improve children's knowledge about dog safety and (2D) efficacy to change how children behave in simulated and actual situations with dogs.

Usability encompasses the ability for children to use the programme independently, without adult supervision and as it is intended. It also includes the acceptability of the programme as a reasonable and appropriate educational tool from children's and parents' perspective and considers patterns of website usage, including frequency of access, time spent on the website during each access and individual mouse (computer)/finger (tablet) movements during each access.

Assessment of relevant cognitive skills will occur via experimental laboratory-based assessment of three constructs: impulse control, perspective taking and attention to details. Assessment of dog safety knowledge will occur via self-report and identification of safe and dangerous situations in photographs. Assessment of behaviour change will occur via a dollhouse simulation task and via a structured interaction with a live dog.

\section{WEBSITE DEVELOPMENT}

\section{Overview}

We will develop a website designed to teach children cognitive skills necessary to be safe with pet dogs. Our goal is to create an entertaining and engaging website that children return to because it is fun but that also helps children develop cognitive skills that are within their developmental capacity to learn and hone, and that will help them be safe with their dogs.

The website will have two primary screens, games and videos. Surprising perhaps, not all website activities will use dog themes. Our goal is to increase children's safety with dogs. If children learn to control their impulses better, and apply that learning when playing with a dog, we will accomplish our objective. The learning might take place through a cognitionbased game without dogs, but that learning will ultimately improve interactions with dogs.

\section{Games}

Young children play, enjoy and learn from educational games. ${ }^{11} 13$ The website will include several games, none requiring literacy. The games will focus in three domains: impulse control, perspective-taking and recognising details.

Games to teach and practice impulse control are important because impulsive behaviour near dogs can upset animals and lead to bites. We will develop games to help children learn, practice and hone impulse control skills. Some will be 'classical' tasks such as Stroop-like (distinguish colour vs name of an object when presented, eg, pictures of orange and green carrots) $^{14}$ and go/no-go (respond when common stimulus appears but restrain for rare stimulus) activities. Others will be novel (throw stick for dog by pulling slingshot-child will throw stick as far as possible three times, then throw a short distance; can child restrain?). Previous work demonstrates the capacity for preschool-aged children to learn these cognitive skills via computer games. ${ }^{15}$

Games to teach and practice perspective-taking will allow children to develop skills to recognise dogs' desires. Dogs differ somewhat, but there are certain signs adults readily interpret 
that children do not. Most adults readily recognise a dog's desire to fetch a ball, for example. Children may not because their perspective-taking skill is still developing and they do not process and understand information from others (people or animals) like adults do. Piaget ${ }^{16}$ recognised this decades ago, and contemporary theory of mind research ${ }^{17}$ suggests perspective-taking skills emerge between ages 3 years and 5 years and continue to develop through middle childhood. ${ }^{18} 19$ To help children develop and hone such skills, we will present games on the topic. For example, we will develop internet-based games offering 'classical' theory of mind scenarios such as false belief (eg, John enters room, hides toy under a hat and leaves; Mom enters room and moves toy from under hat to inside cupboard; John re-enters. Where will John look for the toy?). Research suggests preschool-aged children can learn such perspective-taking abilities with practice, ${ }^{20} 21$ including via computer training. ${ }^{22}$

Finally, we will create innovative perspective-taking games to show children the world from the 'dog's eyes'. Children will see a scene, for example, as a dog would and click one of several choices about what the dog might do next. Right and wrong answers will be tallied. For example, children will view a scene with three items-dog's toy, child's toy, adult's purse-shown from the dog's eye-perspective. Children will click on the dog's choice to retrieve (correct: dog's toy). Later, the game's perspective will change to the child-eye and the identical scene presented to click the preferred object, this time for the child (correct: child's toy).

The last category of games, designed to teach and practice recognition of details, will improve children's ability to notice detailed cues on a dog's body, another key aspect of safety with dogs. Recognising whether a dogs' ears are raised or not, or whether the dogs' eyes are open or shut, can dictate whether to engage with that dog or not. Finer aspects of visual perception, such as contrast sensitivity, perceptual differentiation, and the integration of the perceptual and cognitive systems, develop rapidly through the preschool and early school years. ${ }^{23-25}$ Honing of perceptual skills to recognise and then process subtle differences may help children identify, recognise, and respond to subtle detailed portions of a dog's moods, desires and states. As an example, two drawings of a dog collar will be displayed and children will click the (subtle) differences between the drawings (eg, one has four holes, the other five; one a straight clasp and the other rounded). Other 'difference games' will require detection of aural differences. Aural perception develops rapidly during the preschool years, ${ }^{24}$ and safe engagement with dogs benefits from that development (eg, distinguishing dog's happy and angry barks).

\section{Videos}

Contemporary internet users-especially youth-prefer watching computers rather than reading from them. ${ }^{26}$ We will offer two sets of videos, with all clips purposely brief $(<5 \mathrm{~min})$. First is 'The Dog's View', a unique set of videos showing the world as dogs see it by attaching (or pretending to attach) small cameras to dog collars while they interact with children and culling appropriate segments. Videos will include children behaving assertively with dogs, dogs experiencing children who bother them and dogs engaging in stressful encounters. These videos are expected to be entertaining, but also to teach children the perspective of animals.

Second, we will offer testimonials from children who have experienced dog bites. Extensive research suggests testimonials influence positive health behaviour change. ${ }^{27}$ Created by a videographer who interviews diverse child actors describing circumstances of experiencing a dog bite, our goal will not be to horrify viewers, but to present realistic information that leads viewers to recognise that they are susceptible and vulnerable to bites, that children like them have been bitten by pets assumed to be 'safe' and 'good', and that the 'unthinkable' and 'impossible' actually could occur to them.

\section{Reward system/messaging to parents}

A behavioural reward system will motivate children to (A) spend time on the website, (B) explore the entire website and (C) share lessons with parents. Hearkening classical theory, ${ }^{28}$ behavioural reinforcements are repeatedly shown to improve health behaviour. ${ }^{29}$ Children will receive points for completed activities and use points to achieve higher 'levels'. When children reach certain point levels (purposely arranged for frequent rewards, especially during early stages of usage), they will be rewarded with incentives such as new characters, new opportunities, and printable certificates and prizes.

Besides offering incentives to earn more points and receive awards, the point system is designed to educate and inform parents. When children earn points or achieve new levels, a message will be sent to parents via email or text message. Parental messages will congratulate children and offer parents the opportunity to congratulate (electronically and in person), but also will include targeted information about what children learn. This is an unique aspect of our website. Reaching busy parents to deliver safety messages is challenging. Previous attempts-for example, the 48-page parent manual of The Blue Dog programme-failed, largely because parents do not read nicely prepared but lengthy materials. ${ }^{30}$ Delivering brief congratulatory but also educational messages periodically-one message will be sent to parents for each 15 min children spend on the website-will keep parents closely informed but not inundate them with lengthy material they do not read or process. We recognise reality: today's overextended parents are unlikely to spend time on the internet or elsewhere learning about child dog safety even though they might benefit from doing so. Instead, we will deliver brief succinct messages via technology to parents-messages they likely will see and readand expect that such messages will impact parent cognitions and ultimately behaviour. As an example, we may text, "Congrats! Your child completed level 3 of "Fun \& Games with My Dog Koko". Remind him/her that sleeping dogs should be left alone. Only play w dogs when awake". Although such messaging is novel-we developed the notion for this project-research on adult $^{31}$ and pediatric ${ }^{32}$ health behaviour change, as well as in injury prevention ${ }^{33}$ supports the potential of such message delivery systems.

\section{EVALUATION STUDY}

After the website is developed, we will conduct a repeated measures experiment with pretest and post-test assessments. ${ }^{34}$ Methodological details appear below.

\section{Participants}

Sixty-eight participants ages 4-6 years will be recruited from the UAB Youth Safety Lab Database, which includes over 1600 local families interested in participating in safety-related research. Database families are recruited primarily from local schools and are highly diverse racially, culturally and socioeconomically. We will screen to ensure English fluency, appropriate age range (4-6 years), regular exposure to dogs (at home or homes of friends/family/neighbors), reliable internet access, and 
that children do not have disabilities prohibiting valid participation (eg, blindness, mental retardation). We anticipate a racially diverse ( $\sim 50 \%$ ethnic minority), gender-balanced sample. Participants will provide informed consent (parents) and assent (children) and will be compensated for their time. The study has been reviewed and approved by our university institutional review board.

\section{Protocol}

Following recruitment, families will visit the laboratory, complete consenting and then engage in a battery to assess knowledge and behaviour relevant to dog safety (details below). At the end of the visit, families will be randomly assigned to a group, either the dog website or the control transportation safety website. Following a 2 -week intervention period, families will return to the laboratory to complete the laboratory battery a second time.

\section{Measures}

The following topics will be assessed: demographics, website usability, children's cognitive skills and children's behaviour with dogs. Demographics will be assessed during the baseline (prerandomisation) visit and website usability postintervention. All other measures will be assessed at both visits.

Demographics will be assessed via parent questionnaire. Website usability will be assessed among both groups in two ways. First, families will complete a daily usage diary evaluating website usage (time and frequency), adult assistance to use the website, perceived education and perceived enjoyment. Second, during postintervention visits, we will survey parents concerning perceived usability, educational value, entertainment value, time spent on website and assistance provided to children.

Children's cognitive skills will be assessed preintervention and postintervention using multiple methods. Impulse control will be assessed with neuropsychological and laboratory measures. A brief neuropsychological battery will be constructed using age-appropriate tasks such as Stroop, Go/No-Go and Trails. Behavioural measures will consist of a behavioural battery ${ }^{35}$ including tasks to assess ability to temper fine (Draw-a-Circle) and gross (Walk-a-Line) motor movement, control verbal impulses (Long Speech), and delay gratification for short (Peeking) and long (Pencils) latencies. Other tasks assess decision-making speed (Prize-Choosing) and behavioural surgency (Newspaper Ripping).

Perspective-taking will be assessed by several standard laboratory measures of theory of mind/perspective-taking. We will use vignettes to test first-order ${ }^{36}$ and more advanced second-order ${ }^{37}$ false belief ability. We will test appearance vs reality ability (eg, sponge that looks like a rock; rubber fried eggs ${ }^{38}$ ) and children's ability to infer emotional states via Test of Emotion Comprehension. $^{39}$

Attention to details will be evaluated using a neuropsychological battery including tasks such as WISC-IV (Wechsler Intelligence Scale for Children-IV) ${ }^{40}$ (eg, picture completion) and Test of Visual-Perceptual Skills- $3^{41}$ subtests (eg, visual form constancy, visual figure-ground) and the Children's Embedded Figures Test. $^{42}$

We will assess children's dog safety knowledge in two ways: (A) a basic oral 'quiz' concerning dog safety and (B) a series of photographs showing dogs in various settings/emotional states (eg, angry in cage; playful in park), with children responding whether they would play with each photographed dog. ${ }^{10} 43$

Children's behaviour with dogs will be assessed with two strategies. First, a dollhouse simulation will present children with 10 common scenarios using a dollhouse plus people/dog characters, and ask the child to complete the 'story'. ${ }^{10}$ For example, one reads, "(Child Name) is playing around in the kitchen near (Dog Name's) food. (Dog) comes into the kitchen and sees (Child) near his/her food bowl making him/her upset and start to growl. What happens next?" A single safety score is coded from videotapes using previously developed schemes ${ }^{10}$ and standard protocol (eg, objective definitions, independent coders, inter-rater reliability). Second, a semistructured interaction with a live therapy dog will be used. Developed for previous work, ${ }^{10} 44$ the three-segment, 10-min interaction elicits risk-taking (eg, petting/approaching dog while it 'naps'; choosing to pet dog vs throw ball to it) and inappropriate behaviour (eg, aggression with dog) from children during unstructured, semistructured and structured activities. Behaviour is videotaped and coded.

\section{Interventions}

The dog safety intervention will comprise regular visits to the newly developed dog safety website over a 2 -week period. The comparison group will visit Otto the Auto website, developed by California AAA to promote transportation safety (eg, helmet use, look both ways). Of similar size/scope to the proposed website, Otto the Auto offers numerous games, stories and interactive activities. In both groups, participants will have 2 weeks to use the website regularly (based on similar previous research $\left.{ }^{10}{ }^{45}\right)$. We will remind families to visit the assigned website regularly.

\section{Data analysis plan}

Specific Aim \#1 is to develop an innovative and engaging website to train young children in safety with dogs and will not be addressed analytically. Specific Aim \#2A is to evaluate usability of the website. Descriptive statistics will examine self-reported website usage as well as self-reported perceived education and enjoyment from the website and child assistance needed to use it. $t$ Tests (continuous data) and $\chi^{2}$ tests of association (categorical) will be used to compare the two groups.

Specific Aim \#2B is to evaluate efficacy of the programme to improve relevant cognitive skills in three domains: impulse control, perspective-taking and attention to details. Each construct will be assessed via multiple measures. We will standardise and then aggregate multiple measures for analysis (internal reliability assessed via Cronbach's $\alpha$ and factor analysis/correlation matrices used to develop/verify appropriate aggregates). Following aggregation, we will test group differences by fitting the following linear mixed model:

$$
Y_{\text {ij }}=\beta_{0}+\beta_{1} X_{\text {group }}+\beta_{2} X_{\text {time }}+\beta_{3} X_{\text {group }} \times X_{\text {time }}+\varepsilon_{\text {ij }}
$$

where $Y_{i j}$ is the continuous outcome. Of greatest interest is $\beta_{3}$, the parameter that addresses whether the effect of the intervention differs across time. Specific Aim \#2C is to evaluate the programme's efficacy to improve children's dog safety knowledge. Knowledge will be evaluated through two measures, the 'quiz' and the photographs. We will aggregate the measures if they correlate; otherwise, separate analyses will be conducted. In both cases, we will fit the same model as Specific Aim \#2B. Specific Aim \#2D, to evaluate the programme's efficacy to change children's behaviour, will be evaluated similarly (with dollhouse simulation and live dog measures as dependent variables).

Secondary analyses related to Specific Aim \#2 will test whether the effect of the intervention differs as a function of 
covariates such as age, gender, race/ethnicity, dog ownership/ exposure and website usage patterns.

\section{Retention, missing data and power}

We will implement multiple strategies to retain the sample for the 2 weeks between pretesting and post-testing. These include between-visit contact, collecting multiple methods with which to make contact (phone, email) for parents and family members/friends, reimbursing families for their time and building close rapport via multiple strategies, including a multicultural research staff. Despite these strategies, we anticipate some missing data for multiple reasons, including attrition. Intention-to-treat analyses will be conducted. If significant differences are discovered between completers and noncompleters, multiple imputation will be used.

A power analysis was conducted to determine sample size. Based on previous work, ${ }^{10}$ we assumed change of 1 unit $(\mathrm{SD}=1.5)$ in the dog website condition and 0 units $(\mathrm{SD}=1.5)$ in the comparison condition. Using a two-tailed two-sample $t$ test and assuming $\alpha=.05$, we should have power $>0.70$ to detect that difference with $n=29$ in each group. For the dependent samples pre-post comparison, assuming intergroup correlation of 0.5 , power $>0.95$. Allowing conservatively for $15 \%$ attrition, our proposed sample size of 68 is sufficient to evaluate the website and offer valuable data to inform future study.

\section{CONCLUDING THOUGHTS}

Despite public health risks of paediatric dog bites, empirically supported prevention programmes are few. The proposed website offers several advantages over existing prevention programmes:

- It can be widely disseminated via the internet. Most American children have internet access at home and school, and global access via internet is increasingly plausible.

- It will be technologically sophisticated, using the power of today's internet to deliver messages to children.

- It will be based on scientific theory and empirical data.

- It will target multiple aspects of safety with dogs-not just rote learning of rules that may not alter behaviour.

- It will be entertaining and visually appealing. We expect children to enjoy the website and return often.

- It will connect to parents via technology in a novel, pragmatic and theory-driven manner.

- It will be modifiable. In response to empirical evaluation, user feedback, and increasing scientific and applied knowledge, we can update, improve and expand the website.

- Beyond our foremost goal of improving child health, the programme will improve animal health. Children who play calmly and appropriately with pet dogs have happier and healthier dogs. Dogs exposed to rough, inappropriate or bothersome behaviour by children are stressed. In extreme situations when dogs bite children, they may be euthanised. This programme may result in reduced pet euthanasia.

- Our programme corresponds with movements to translate basic scientific knowledge into practice, to use technology to communicate health messages, and to implement innovative and novel ways of retaining and improving human health. ${ }^{46-48}$

The initial evaluation study described here will examine usability and feasibility as well as changes in children's knowledge and behaviour. If successful, we expect it will lead to a larger-scale randomised controlled trial that evaluates efficacy of the website compared with active control groups with assessment of knowledge and behaviour retention over time. If this programme proves successful, it holds excellent potential for broad global dissemination.

Acknowledgements Thanks to the Digital Artefacts team for website development and IT support. Thanks to Anna Johnston, Jiabin Shen and the UAB Youth Safety Lab for anticipated data collection and processing. The research reported in this publication was supported by the Eunice Kennedy Shriver National Institute of Child Health and Human Development of the National Institutes of Health under Award Number R21HD075960. The content is solely the responsibility of the authors and does not necessarily represent the official views of the National Institutes of Health.

Competing interests None.

Ethics approval University of Alabama at Birmingham IRB.

Provenance and peer review Not commissioned; internally peer reviewed.

\section{REFERENCES}

1 Centers for Disease Control [CDC]. Dog bite: Fact sheet. 2008. Retrieved 4/29/14 from http://www.cdc.gov/homeandrecreationalsafety/dog-bites/index.html

2 Centers for Disease Control [CDC]. Nonfatal dog bite-related injuries treated in hospital emergency departments-US 2001. MMWR 2003;5226:605-10.

3 Kaye AE, Belz JM, Kirschner RE. Pediatric dog bite injuries: a 5-year review of the experience at The Children's Hospital of Philadelphia. Plast Reconstr Surg 2009;124:551-8.

4 Morrongiello BA, Marlenga B, Berg R, et al. A new approach to understanding pediatric farm injuries. Soc Sci Med 2007;65:1364-71.

5 Jalongo MR. Beyond a pets theme: Teaching young children to interact safely with dogs. Early Child Educ J 2008;36:39-45.

6 Chapman S, Cornwall J, Righetti J, et al. Preventing dog bites in children: Randomised controlled trial of an educational intervention. BMJ 2000;320:1512-13

7 Spiegel IB. A pilot study to evaluate an elementary school-based dog bite prevention program. Anthrozoos 2000;13:164-73.

8 Wilson F, Dwyer F, Bennett PC. Prevention of dog bites: Evaluation of a brief educational intervention program for children. J Community Psychol 2003;31:75-86.

9 Meints K, de Keuster T. Brief report: don't kiss a sleeping dog: The first assessment of "The Blue Dog" bite prevention program. J Pediatr Psychol 2009;34:1084-90.

10 Schwebel DC, Morrongiello BA, Davis AL, et al. The Blue Dog: evaluation of an interactive software program to teach young children how to interact safely with dogs. J Pediatr Psychol 2012;37:272-81.

11 Zevenbergen $\mathrm{R}$, Logan $\mathrm{H}$. Computer use by preschool children: Rethinking practice as digital natives come to preschool. Aust J Early Child 2008;33:37-44.

12 Alikhan N. Integrating technology and games to engage and motivate students in the 21 st Century classroom. Presented at the 10th Annual ISNA Education Forum; Chicago, IL, 2009. Retrieved 4/29/14 from http://www.aldeenfoundation.org/ GamestoEngageMotivateStudentsinthe21stCenturyClassroom.pdf

13 Rideout VJ, Vandewater EA, Wartella EA. Zero to six: electronic media in the lives of infants, toddlers and preschoolers. Menlo Park, CA: Henry J Kaiser Family Foundation, 2003.

14 Prevor MB, Diamond A. Color-object interference in young children: A Stroop effect in children 31/2-61/2 years old. Cogn Dev 2005;20:256-78.

15 Thorell LB, Lindqvist $S$, Nutley SB, et al. Training and transfer effects of executive functions in preschool children. Dev Sci 2009:12:106-13.

16 Piaget J. The language and thought of the child. New York: Humanities Press, 1959.

17 Wellman HM, Cross D, Watson J. Meta-analysis of theory-of-mind development: The truth about false belief. Child Dev 2001;72:655-84.

18 Ketelaars MP, van Weerdenburg M, Verhoeven $L$, et al. Dynamics of the theory of mind construct: A developmental perspective. Eur J Dev Psychol 2010;7:85-103.

19 Miller SA. Children's understanding of second-order mental states. Psychol Bull 2009;135:749-73.

20 Lu H, Su Y, Wang Q. Talking about others facilitates theory of mind in Chinese preschoolers. Dev Psychol 2008;44:1726-36.

21 Ornaghi V, Brockmeier J, Gavazzi IG. The role of language games in children's understanding of mental states: A training study. J Cogn Dev 2011;12:239-59.

22 Swettenham J. Can children be taught to understand false belief using computers? J Child Psychol Psychiatry 1996;37:157-65.

23 Adams RJ, Courage ML. Using a single test to measure human contrast sensitivity from early childhood to maturity. Vision Res 2002;42:1205-10.

24 Bornstein MH, Arterberry ME, Mash C. Perceptual development. In: Bornstein MH, Lamb ME, eds. Developmental science: an advanced textbook. 5th edn. Mahwah, NJ: Erlbaum, 2005:283-325.

25 Vlamings PHJM, Jonkman LM, Kemner C. An eye for detail: An event-related potential study of the rapid processing of fearful facial expressions in children. Child Dev 2010;81:1304-19. 


\section{Study protocol}

26 Ariño M. Content regulation and new media: A case study of online video portals. Commun Strateg 2007;66:115-35.

27 Meisel ZF, Karlawish J. Narratives vs evidence-based medicine-and, not or. JAMA 2011:306:2022-3.

28 Skinner BF. The behavior of organisms. New York: Appleton-Century-Crofts, 1938.

29 Suls JM, Davidson KW, Kaplan RM. Handbook of health psychology and behavioral medicine. New York: Guilford, 2010.

30 Morrongiello BA, Schwebel DC, Stewart J, et al. Examining parents' behaviors and supervision of their children in the presence of an unfamiliar dog: does The Blue Dog intervention improve parent practices? Acc Anal Prev 2013;54:108-13.

31 Fjeldsoe BS, Marshall AL, Miller YD. Behavior change interventions delivered by mobile telephone short-message service. Am J Prev Med 2009;36:165-73.

32 Militello LK, Kelly SA, Melnyk BM. Systematic review of text-messaging interventions to promote healthy behaviors in pediatric and adolescent populations: implications for clinical practice and research. Worldviews Evid Based Nurs 2012;9:66-77.

33 Ricketts M, Shanteau J, McSpadden B, et al. Using stories to battle unintentional injuries: Narratives in safety and health communication. Soc Sci Med 2010;70:1441-9.

34 Campbell DT, Stanley JC. Experimental and quasi-experimental designs for research. Chicago: Rand McNally, 1963.

35 Schwebel DC. Temperamental risk factors for children's unintentional injury: the role of impulsivity and inhibitory control. Pers Individ Dif 2004;37:567-78.

36 Perner J, Wimmer H. John thinks that Mary this that-attribution of 2nd order beliefs by 5-year-old to 10-year-old children. J Exp Child Psychol 1985;39:437-71.
37 Sullivan K, Zaitchik D, Tager-Flusberg H. Preschoolers can attribute second-order beliefs. Dev Psychol 1994;30:395-402.

38 Flavell J, Flavell E, Green F. Development of the appearance-reality distinction. Cogn Dev 1983;15:95-120.

39 Pons F, Harris P. Test of emotion comprehension. Oxford, UK: University of Oxford, 2000.

40 Wechsler D. Wechsler intelligence scale for children. 4th edn. San Antonio, TX: Harcourt Assessment Inc, 2003.

41 Martin NA. Test of visual-perceptual skills non-motor. 3rd edn. Torrance, CA: Western Psychological Services, 2006.

42 Witkin $\mathrm{HA}$, Oltman PK, Raskin $\mathrm{E}$, et al. A manual for the embedded figures test. Palo Alto, CA: Consulting Psychologists Press, 1971.

43 Shen J, Li S, Xiang H, et al. Dog safety in rural China: children's sources of safety information and effect on knowledge attitudes and practices. Acc Anal Prev 2013;59:164-9.

44 Davis AL, Schwebel DC, Morrongiello BA, et al. Dog bite risk: an assessment of child temperament and child-dog interactions. Int J Environ Res Public Health 2012;9:3002-13.

45 Morrongiello BA, Schwebel DC, Bell M, et al. An evaluation of The Great Escape: can a computer game improve young children's fire safety knowledge and behaviors? Health Psychol 2012;31:496-502.

46 Woolf SH. The meaning of translational research and why it matters. JAMA 2008:299:211-13.

47 Zerhouni E. The NIH roadmap. Science 2003;302:63-72.

48 Zerhouni EA. Translation and clinical science-time for a new vision. New Eng/ J Med 2005;353:1621-3. 